\title{
Las posibilidades del Consejo Genético en Atención Primaria
}

\author{
José M. ${ }^{a}$ Alonso Gordo ${ }^{a}, M^{a}{ }^{a}$ del Carmen Sánchez González ${ }^{a}$, \\ Natacha Hernández Pérez ${ }^{b}$, M. $^{a}$ José Calvo Orduña ${ }^{c}$
}

\author{
${ }^{a}$ Médico de Familia. \\ ${ }^{b}$ Residente de Medicina de \\ Familia y Comunitaria. \\ c Diplomada Universitaria en \\ Enfermería. \\ Centro de Salud Guadalajara- \\ Sur. Servicio de Salud \\ de Castilla-La Mancha. \\ Guadalajara (España) \\ Dirección para \\ correspondencia: \\ José M. ${ }^{a}$ Alonso Gordo. \\ Correo electrónico: \\ jmalonso@sescam.org
}

Recibido el 27 de marzo de 2014.

Aceptado para su publicación el 13 de mayo de 2014.

\section{RESUMEN}

El Consejo genético ayuda a los pacientes en la toma de decisiones en aspectos relacionados con la posibilidad de padecer o transmitir una enfermedad determinada genéticamente. El conocimiento del mapa del genoma humano y los desarrollos tecnológicos hacen posible detectar alteraciones cromosómicas, enfermedades con transmisión mendeliana, defectos metabólicos, marcadores de múltiples enfermedades, que nos permiten adoptar medidas preventivas para mejorar nuestro estado de salud o el de nuestros descendientes.

En las consultas de Atención Primaria disponemos de múltiples oportunidades para poder detectar e incluso prevenir enfermedades con componente hereditario, no obstante, en la mayoría de las ocasiones será necesario derivar al escalón especializado para la realización de las pruebas necesarias y la aplicación del consejo genético.

Además de los componentes técnicos y metodológicos, existen múltiples aspectos personales que obligan a ser especialmente cuidadosos con los aspectos éticos en la aplicación del consejo genético: confidencialidad, derecho a la información propia o de terceros, consecuencias legales, psicológicas, sociales o laborales, etc.

Pretendemos con el presente artículo colaborar a que los profesionales de Atención Primaria conozcan mejor las posibilidades del consejo genético y, en consecuencia, puedan informar y orientar adecuadamente a sus pacientes potencialmente afectos de alguna enfermedad con componente genético o hereditario.

Palabras clave: Consejo genético. Estudios genéticos. Enfermedades hereditarias. Atención Primaria.

\section{ABSTRACT}

The possibilities of genetic counselling in primary healthcare

Genetic counselling helps patients to take decisions in matters related to the possibility of suffering from or transmitting a certain disease genetically. Knowledge of the map of the human genome and technological developments make it possible to detect chromosomal alterations, Mendelian disease transmission, metabolic defects, markers of multiple diseases, which allow us to adopt preventive measures in order to improve our state of health, or of our descendants. In primary care consultations, we have at our disposal manifold opportunities to detect and even prevent diseases with a hereditary component although, on most occasions, referral to a specialized level will be necessary in order to carry out necessary tests and apply genetic counselling. In addition to technical and methodological components, there are multiple personal aspects which oblige us to be take special care of ethical issues involved in genetic counselling; confidentiality, right to proprietary or third party information, legal, psychological, social or workrelated consequences, etc.

In this article, it is our aim to collaborate so that primary care professionals are made more aware of the possibilities of genetic counselling and thus be able to inform and give proper guidance to their patients who are potentially affected by a disease with a genetic or hereditary component.

Key words: Genetic counselling. Genetic studies. Hereditary diseases. Primary healthcare. 


\section{INTRODUCCIÓN}

El Consejo Genético (CG) se puede definir como el proceso por el que se informa a los pacientes o familiares del riesgo de padecer una enfermedad hereditaria, de la posibilidad de trasmitirlo a las siguientes generaciones, de las medidas preventivas o terapéuticas que se pueden realizar y de la posibilidad de realizar un test genético para su detección ${ }^{1}$. En nuestro país es un servicio incluido, de modo genérico, en la planificación familiar por parte de nuestro sistema sanitario ${ }^{2}$.

Aunque la mayoría de las actuaciones relacionadas con esta prestación competen a la atención espe- retardo mental no explicado, edad materna avanzada, exposición a teratógenos, consanguinidad, esterilidad o infertilidad y abortos espontáneos de repetición ${ }^{7-9}$. Recientemente, dos hechos han incrementado la necesidad de tener una adecuada formación en temas relacionados con la genética, la elaboración del mapa del genoma humano y el interés creciente por las denominadas "enfermedades raras" (hasta 8.000 patologías de las que el 80 $\%$ pueden tener un origen genético), que pueden afectar hasta a tres millones de personas en nuestro país ${ }^{10}$. En la tabla 1 podemos ver la incidencia estimada de algunas enfermedades hereditarias ${ }^{11}$.

\begin{tabular}{l|l}
\hline Enfermedad & Incidencia \\
\hline Distrofia muscular de Becker & $1 / 18.000$ nacidos \\
Fibrosis quística & $1 / 2.500$ \\
Distrofia muscular de Duchenne & $1 / 4.000$ varones \\
Cáncer de mama familiar & $4-8 / 1.000$ mujeres \\
Poliposis adenomatosa de colon & $1-8 / 13.500$ \\
Síndrome de cromosoma X frágil & $1-5 / 5.000$ varones \\
Ataxia de Friedrich & $1-2 / 100.000$ \\
Hemofilia A & $1 / 5.000$ varones \\
Hemofilia B & $1 / 25.000$ varones \\
Hemocromatosis & $5 / 1000$ \\
Neuropatías sensitivomotoras & $10-40 / 10.000$ \\
Corea de Huntington & $0,65 / 100.000$ \\
Distrofia miotónica & $1 / 8.000$ \\
Síndrome de Prader Willi & $1 / 15-20.000$ \\
Atrofia muscular espinal & $1 / 10.000$ \\
\hline
\end{tabular}

Tabla 1. Incidencia estimada o prevalencia de algunas enfermedades hereditarias ${ }^{11}$

cializada, con relativa frecuencia se plantean en la consulta del médico de Atención Primaria (AP) cuestiones referentes a las posibilidades de sufrir un cáncer $u$ otra enfermedad con carácter genéti$\mathrm{co}$, o a la necesidad de realizar alguna prueba para conocer el riesgo de padecerla ${ }^{3-6}$. También algunas intervenciones, como veremos más adelante, están en el ámbito de actuación de los profesionales de AP.

Existe una serie de indicaciones habituales para la realización del CG entre las que están: enfermedad genética conocida o sospechada en un paciente o su familia, diagnóstico presintomático y antenatal de afecciones genéticas, defectos de nacimiento,
Siendo la AP el lugar donde habitualmente se hace la primera consulta, y aunque en muchas ocasiones la solución final vendrá dada tras la derivación a una consulta especializada, el profesional de aquel ámbito debe tener unos conocimientos básicos que orienten adecuadamente el problema. En este sentido, la información incompleta o equivocada que podamos ofrecer, así como la omisión de pruebas recomendables, puede tener consecuencias indeseables para nuestros pacientes. Por el contrario, la indicación de pruebas o consultas innecesarias puede dar lugar a una utilización indebida de recursos y, en muchas ocasiones, a la creación de alarmas y etiquetados que solo provocan ansiedad, sin beneficio alguno para la salud. 


\section{GENÉTICA: UN RECORDATORIO}

Es conocido que la especie humana presenta un patrimonio genético constituido por 23 pares de cromosomas, de los cuales 22 son los autosomas y el par restante los cromosomas sexuales (XX para la mujer, $X Y$ para el varón). Las alteraciones en los cromosomas pueden ser debidas al número (aneuploidías) o a la estructura (translocaciones, deleciones, etc.). En todo caso, las enfermedades genéticas pueden ser autosómicas o ligadas al sexo, además de las enfermedades mitocondriales que tienen unas características especiales y están ligadas casi exclusivamente al ovocito y, por tanto, a la herencia materna ${ }^{6,11,12}$.

A su vez, las enfermedades con componente hereditario pueden tener un carácter monogénico, si vienen determinadas por un solo gen, o poligénico, en las que intervienen varios genes en su aparición y manifestaciones ${ }^{11,12}$. Asimismo, la penetrancia nos indica la capacidad de aparecer con mayor o menor frecuencia en generaciones sucesivas, mientras que la expresividad nos informa sobre la capacidad de manifestarse clínicamente de modo más o menos florido. Por tanto, una enfermedad determinada puede tener una baja penetrancia, pero una alta expresividad clínica, siendo, por ejemplo, el síndrome de Down o la neurofibromatosis modelos de la alta variabilidad que pueden tener en sus manifestaciones clínicas.

Se considera que un gen es dominante cuando siempre se expresa en el individuo, incluso cuando en él solo está presente una copia (afectada) de unos de los progenitores, dando lugar a una enfermedad autosómica dominante. Por su parte, un gen es autosómico recesivo si para que se exprese se necesita que confluyan, combinadamente, genes afectados de ambos progenitores, dando lugar a una enfermedad autosómica recesiva, mientras que, si solo está afectado el gen de uno de los progenitores, la persona receptora no estará afectada, pero será portadora ${ }^{12,13}$. En la figura adjunta (figura 1) y a modo de recordatorio, se puede ver el modelo simplificado de herencia de un carácter autosómico recesivo. En las tablas 2, 3 y 4 se presentan algunas de las enfermedades con dicho carácter autosómico recesivo, dominante y ligadas al sexo, respectivamente ${ }^{14}$.

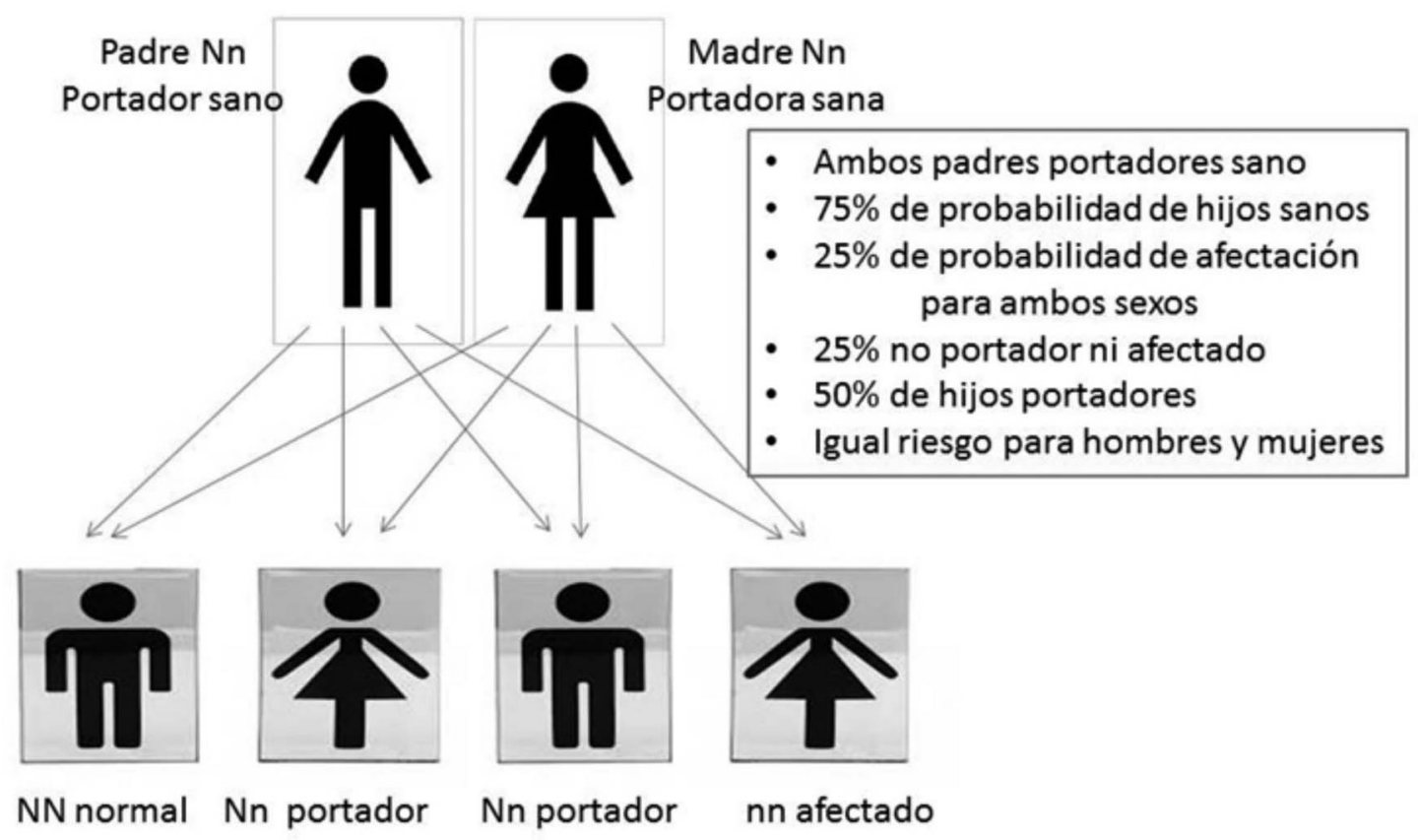

Figura 1. Modelo de herencia autosómico recesivo 


\section{Síndrome de Marfan \\ Osteogénesis imperfecta \\ Neurofibromatosis \\ Braquidactilia \\ Retinoblastoma \\ Esclerosis tuberosa \\ Poliposis múltiple del colon}

Tabla 2. Principales enfermedades autosómicas dominantes ${ }^{14}$

\begin{tabular}{ll}
\hline Fibrosis quística & Hipofosfatemia familiar \\
Beta-talasemia & Anemia de Fanconi \\
Atrofia muscular espinal & Ataxia de Friedrich \\
Enfermedad de Tay-Sach & Déficit acetil-coA \\
Isoinmunización Rh & Acidemia metilmalónica \\
Enfermedad de Gaucher & Déficit ornitin-transcarbamilasa \\
Adrenoleucodistrofia & Déficit piruvatodeshidrogenasa \\
Factor V Leiden & Riñón poliquístico \\
\hline
\end{tabular}

Tabla 3. Principales enfermedades autosómicas recesivas ${ }^{14}$

\begin{tabular}{ll}
\hline Distrofia muscular de Duchenne y Becker & Hemofilia \\
Síndrome X frágil & Retraso mental ligado al X \\
Síndrome de Wiskott-Aldrich & Síndrome Charcot-Marie Tooth \\
Hidrocefalia & Enfermedad granulomatosa \\
Agammaglobulinemia & Enfermedad de Anderson-Fabry \\
Autismo & Síndrome de Barth \\
Displasia ectodérmica hipohidrótica & Incontinencia pigmenti \\
Enfermedad de Kennedy & Síndrome de Lowe \\
Retinitis pigmentosa & Retinosquisis \\
Raquitismo Vitamina D resistente & \\
\hline
\end{tabular}

Tabla 4. Algunas enfermedades ligadas al sexo ${ }^{14}$

Son enfermedades ligadas al sexo aquellas cuyo gen radica en uno de los cromosomas sexuales, $\mathrm{X}$ o $\mathrm{Y}$. Por lo general, el gen de la mayoría de las enfermedades ligadas al sexo está localizado en el cromosoma $\mathrm{X}$ y suelen tener carácter recesivo. Por este motivo, las mujeres que tienen otro cromosoma $X$ compensador no están afectadas y solo transmiten la enfermedad, mientras que los varones, carentes del $2^{\circ}$ cromosoma $X$, padecen y trasmiten la enfermedad.

\section{OPORTUNIDADES DE PARTICIPACIÓN EN EL CONSEJO GENÉTICO EN AP}

Bajo el punto de vista de los profesionales de AP, podemos destacar varios momentos en los que es posible intervenir para realizar actuaciones 0 responder a cuestiones relacionadas con el CG. Aunque en muchos casos la intervención no sea de nuestra competencia directa, las recordaremos con el fin de mejorar nuestro conocimiento y posi- 
bilidades de asesoramiento. Incluimos, asimismo, algunas prácticas que, si bien no se pueden considerar estrictamente como CG, sí que tienen un carácter y finalidad "eugenésicos".

\section{Asesoramiento preconcepcional}

Los profesionales de AP tenemos al menos cuatro oportunidades de intervenir antes de la concepción para evitar posibles problemas posteriores en la descendencia:

a) Promoviendo la ingesta de ácido fólico preconcepcional. Según acuerdo mayoritario, en todas las mujeres con un embarazo planificado o subsidiarias de quedar embarazadas, al menos desde un mes antes hasta dos meses después, para evitar los defectos del tubo neural. La recomendación es más fuerte y las dosis más elevadas para las pacientes con antecedentes personales o familiares de dicho defecto, uso de anticonvulsivantes, obesidad, diabetes o historia de mutaciones enzimáticas relacionadas con el ácido fólico ${ }^{15}$.

b) Recogiendo la historia familiar de ambos miembros de la pareja para identificar los riesgos de presentar una enfermedad genéticamente determinada.

c) Identificando y previniendo en lo posible aquellas situaciones de alto riesgo para la concepción de un embrión con potenciales riesgos para la salud como edad materna avanzada, exposición a teratógenos, historia de abortos espontáneos de repetición, consumo de sustancias tóxicas, etc.

d) Identificando a aquellas parejas con problemas de fertilidad o esterilidad con el fin de poder realizar un estudio adecuado y poder planificar y conseguir un embarazo con garantías y a una edad fisiológicamente aceptable ${ }^{9}$.

En los cuatro casos estamos actuando en el terreno de la prevención primaria, siempre de elección frente a los otros niveles de prevención menos deseables.

\section{Actuación en periodo preimplantacional}

Aunque no es probable que un profesional de AP se vea implicado en este proceso, se puede disponer de la información suficiente que permita realizar una orientación adecuada, independientemente de otros condicionantes respetables, morales o religiosos.

En la fase embrionaria precoz y antes de la implantación fisiológica o tras una fertilización in vitro, se pueden realizar pruebas diagnósticas que nos permitan identificar enfermedades o condiciones favorables para algún tipo de trastorno genéticamente determinado. Es una técnica diagnóstica que permite (obteniendo una célula en la fase de mórula o blástula) seleccionar los embriones genética y cromosómicamente normales antes de implantarlos en el útero materno ${ }^{1}$. Se reduce así el riesgo de transmitir una enfermedad genética familiar grave $y$, por consiguiente, tener que tomar la decisión de un posible aborto terapéutico ${ }^{14}$. Otra opción es la posibilidad de obtención de hijos como potenciales donantes de células madre para hermanos enfer$\operatorname{mos}^{14,16,17}$.

Así, es posible realizar diversas actuaciones en este sentido, como son la determinación del sexo fetal, el diagnóstico de enfermedades hereditarias graves de aparición temprana y no susceptible de tratamiento curativo, o la detección de alteraciones cromosómicas o de otra índole que puedan comprometer la viabilidad del preembrión.

En España, la Comisión Nacional de Reproducción Humana Asistida es el órgano colegiado, de carácter permanente y consultivo, dirigido a asesorar y orientar en esta materia. A su vez, las referencias obligadas son el Convenio de Oviedo, relativo a los derechos humanos y a la biomedicina ${ }^{18}$, la Ley de Reproducción Humana asistida ${ }^{19}$ y la Ley de Investigación Biomédica ${ }^{20}(\mathrm{LIB})$. Dicha ley señala en su artículo 45 los principios rectores específicos, como son la accesibilidad y equidad, protección de datos, gratuidad, el consentimiento y la calidad de los datos. Por supuesto, no es utilizable el uso del diagnóstico preconcepcional para seleccionar características no relacionadas con enfermedad como altura, peso, inteligencia, rasgos de personalidad, comportamiento, o elección arbitraria del sexo $^{19,21}$. La Ley también establece que el CG se debe realizar antes y después de la realización de las pruebas, por personal cualificado e integrado en un equipo multidisciplinar.

\section{Actuaciones posimplantacionales}

Están constituidas por una serie de técnicas que permiten predecir el riesgo de que el feto sea portador de alguna anomalía importante, diagnosticar alguna de dichas anomalías o establecer medidas 
preventivas para evitar importantes daños fetales. Entre estas técnicas se encuentran las siguientes, algunas de las cuales se utilizan de modo habitual en el seguimiento de embarazos normales y otras únicamente en casos excepcionales:

- Asesoramiento para evitar posibles efectos teratógenos o nocivos para la salud del embrión: exposición a radiaciones, fármacos, agentes infecciosos, etc. Este es un terreno inevitablemente unido al trabajo habitual en AP y en el que nuestro papel es fundamental. Para los fármacos, las categorías establecidas por la FDA, de referencia obligada en nuestra actuación, marcadas con las letras A, B, C, D y X representan todo el espectro desde los fármacos seguros hasta los que están totalmente contraindicados ${ }^{22}$.

- Screening metabólico en sangre materna: alfafetoproteina, gonadotropina coriónica humana y otras. Teniendo en cuenta que solo nos dan una probabilidad de afectación (por ejemplo, por el síndrome de Down), pero para confirmar el diagnóstico se debe realizar una técnica invasiva con sus riesgos inherentes para el feto o para la madre. Así mismo, los padres deben tener claro los pasos siguientes a un posible resultado positivo, pues de otro modo podrían generar problemas importantes de angustia y sensación de culpabilidad ${ }^{1}$.

- Técnicas de imagen: ecografías de alta resolución, 3D, Doppler color, etc., con indicación sobre todo para la medición de la traslucencia nucal y su relación con algunas trisomías.

- Técnicas invasivas: amniocentesis, funiculocentesis, biopsia corial, cultivo de amniocitos, etc. Nos pueden informar sobre el sexo fetal, anomalías cromosómicas, enfermedades mendelianas, enfermedades metabólicas, etc.

En la tabla 5 podemos ver el riesgo de tener un hijo afectado por alguna anomalía cromosómica en función de la edad ${ }^{14}$.

\begin{tabular}{ll}
\hline Riesgo & Edad \\
\hline $1 / 385$ & a los 30 años \\
$1 / 179$ & a los 35 años \\
$1 / 63$ & a los 40 años \\
$1 / 19$ & a los 45 años \\
\hline
\end{tabular}

Tabla 5. Riesgo de tener un niño afectado de alguna anomalía cromosómica ${ }^{14}$

\section{Diagnóstico neonatal}

En la mayoría de los países occidentales están vigentes programas de detección precoz neonatal de un amplio, aunque variable, número de enfermedades mediante la denominada "prueba del talón". En nuestro país la $\mathrm{LIB}^{20}$ se refiere a los "programa de determinantes genéticos, para los cuales una intervención médica precoz pudiera conducir a la eliminación o reducción de la mortalidad, morbilidad o discapacidades asociadas a tales determinantes". También establece que "las autoridades sanitarias determinarán, basándose en criterios objetivos, la pertinencia del cribado genético en atención a las enfermedades a prevenir o tratar. Velarán, asimismo, por que se garantice el acceso universal y equitativo de la población para la cual está indicado el cribado".

Pero, sucede que, por un lado, hace escasos años solo a un $20 \%$ de los recién nacidos españoles se le realizaban estas pruebas de cribado y, por otro, los niños acceden a más o menos detecciones dependiendo del año o lugar del nacimiento ${ }^{23}$. Se da así la paradoja de que dos hermanos nacidos en distinto año o en distinta comunidad autónoma pueden ser sometidos a distintos tipos de detecciones, siendo muy difícil de justificar ante los padres esta diferencia cuando en uno de los casos se haya dejado de detectar una posible anomalía. Por su parte, a los propios padres se les pueden plantear problemas importantes de conciencia y de coste/oportunidad, en función de su potencial económico, ante la amplia oferta privada existente en un tema tan sensible para ellos.

El cribado básico o tradicional detecta cinco enfermedades: hipotiroidismo congénito, fenilcetonuria, hiperplasia suprarrenal congénita, fibrosis quística y hemoglobinopatías. Pero solo el hipotiroidismo congénito y la hiperfenilalaninemia eran detectados por todos los servicios sanitarios autonómicos ${ }^{23}$. Otros programas nacionales y europeos incluyen la galactosemia, homocisteinuria, tirosinemia y hasta varias decenas en los cribados neonatales ampliados y/o de oferta privada.

En este sentido, la Ley de Salud Pública establecía en su artículo 6 "una cartera de servicios básica y común en el ámbito de la salud pública, que incluirá un calendario único de vacunación y una oferta única de cribados poblaciones" y recomendaba la elaboración de "la lista de acciones preventivas comunes que reúnan los criterios para ser implantados en todo el territorio"24. 
Afortunadamente, y de modo reciente, el Consejo Interterritorial de Salud ha establecido siete enfermedades que, a partir de ahora, deberán formar parte del cribado neonatal obligatorio: fenilcetonuria, hipotiroidismo congénito, fibrosis quística, déficit de acil Coa cadena media (MCADD), déficit de acil Coa cadena larga (LCHADD), acidemia glutárica y anemia falciforme ${ }^{25}$ (tabla 6). Se mantienen programas piloto para la enfermedad de la orina con olor a jarabe de arce, acidemia isovalérica y homocisteinuria, y se valora la futura inclusión de la hiperplasia suprarrenal congénita, galactosemia y déficit de biotinidasa ${ }^{25}$. De momento, cada Comunidad Autónoma establece las pruebas incluidas en su cartera de servicios y cada equipo de pediatría es el responsable de su aplicación.

\begin{tabular}{ll}
\hline Enfermedad & Tasa \\
\hline Hipotiroidismo & $1 / 2.360$ \\
Fenilcetonuria & $1 / 8.401$ \\
Fibrosis quística & $1 / 4.350$ \\
Deficiencia MCADD & $1 / 23.401$ \\
Deficiencia LCHADD & $1 / 198.554$ \\
Acidemia glutárica I & $1 / 85.094$ \\
Anemia falciforme & $1 / 5.571$ \\
\hline
\end{tabular}

Tabla 6. Tasas de detección de las enfermedades propuestas para cribado por el Consejo Interterritorial de Salud ${ }^{25}$

\section{Búsqueda de portadores sanos}

En determinadas ocasiones se nos puede plantear en la consulta de AP la demanda de pruebas para conocer si una persona sana es portadora de un determinado gen ligado a una enfermedad o a un mayor riesgo de enfermar. Con ello podemos identificar a las personas con una mutación genética para un desorden heredado en forma autosómica recesiva o recesiva ligada al sexo, ya que las enfermedades con patrón dominante tienen manifestaciones clínicas (aunque no siempre son evidentes), y no hay portadores sino enfermos.

En las tablas 2, 3 y 4 hemos recogido la relación de enfermedades más habituales con patrón de herencia autosómico recesivo, dominante o ligado al sexo, y en la tabla 7 algunos ejemplos de trastornos genéticos susceptibles de intervención y su mecanismo de transmisión ${ }^{5}$.

\section{Diagnóstico presintomático}

En este caso nos ocupamos de la realización de pruebas de predicción, las cuales se ofrecen a las personas sin síntomas que, basadas en su historia familiar, están en alto grado de riesgo de desarrollar un desorden. La Academia Americana de Médicos de Familia ha recomendado pautas específicas para cuando se van a realizar pruebas genéticas de predicción ${ }^{21}$, incluyendo:

- Obtener previamente una historia familiar exacta con diagnóstico confirmado.

- Proveer información acerca de la historia natural de la afección.

- Analizar previamente el valor predictivo de la prueba, su exactitud técnica y el significado de un resultado positivo o negativo.

- Explorar los motivos del paciente para someterse a la prueba, el impacto potencial de realizar la prueba y del riesgo de conocer el paso de una mutación a un hijo.

- Discutir problemas referentes a la confidencialidad, al riesgo de desempleo e incluso de discriminación de seguros.

Los trastornos genéticos más habituales, susceptibles de intervención, como los recogidos en la tabla 7 ya se llevan a cabo en múltiples centros de nuestro país ${ }^{11}$.

También la Agencia de Evaluación de Tecnologías Sanitarias ha especificado las condiciones que debe reunir una prueba genética para la incorporación a la oferta asistencial de un centro: metodológicas, de calidad, criterios de inclusión y exclusión, finalidad, eficiencia, beneficios en salud, protección de datos, adecuado consentimiento informado, etcétera ${ }^{16}$.

\section{El riesgo de cáncer}

El CG relacionado con el cáncer es una herramienta que nos permite asesorar a las personas que nos visitan acerca del riesgo que tienen de presentar cáncer, las posibilidades de transmitirlo a la descendencia y las medidas existentes para el diagnóstico temprano y/o la prevención de estos cánceres $^{26}$.

En el caso del cáncer, las pruebas de cribado genético solo se deben realizar a individuos cuyos antecedentes familiares o personales son altamente indicativos de un síndrome de cáncer hereditario (tabla 8) y siempre que la realización de la prueba nos permita adoptar precozmente medidas (realmente disponibles) que mejoren el pronóstico en 


\begin{tabular}{ll}
\hline Trastorno & Herencia \\
\hline Factor V de Leyden & Autosómica Dominante \\
Hemofilia A y B & Ligada al sexo \\
Déficit de Glucosa-6-fosfato DH & Ligada al sexo \\
Síndrome de Marfan & Autosómica Dominante \\
Síndrome de QT largo & Autosómica Dominante \\
Miocardiopatía hipertrófica & Autosómica Dominante \\
Fiebre mediterránea familiar & Autosómica Recesiva \\
Hemocromatosis & Autosómica Recesiva \\
Déficit de alfa-1-antitripsina & Autosómica Recesiva \\
Hipertensión pulmonar primaria & Autosómica Dominante \\
Enfermedad poliquística renal & Autosómica Dominante \\
Diabetes insípida nefrógena & Ligada al sexo, Autosómica Recesiva \\
Neoplasia endocrina múltiple & Autosómica Dominante \\
Síndrome de Kallman & Ligada al sexo \\
Enfermedad de Wilson & Autosómica Recesiva \\
Parálisis hiperpotasémica periódica & Autosómica Dominante \\
Distrofia muscular de Duchenne y Becker & Ligada al sexo \\
Adrenoleucodistrofia & Ligada al sexo \\
\hline
\end{tabular}

Tabla 7. Ejemplos de trastornos genéticos susceptibles de intervención ${ }^{5}$

mayor grado que si se espera al momento en que es posible el cribado poblacional o el diagnóstico clínico. De otro modo, se producirá una situación de incremento de angustia sin beneficio alguno para la salud y la calidad de vida. También hay que tener en cuenta e informar adecuadamente que la negatividad de una prueba no exime del riesgo de sufrir enfermedades de aparición esporádica como sucede, por ejemplo, en el cáncer de colon ${ }^{21}$.

Debemos recordar que cerca de un $25 \%$ de los cánceres tienen una cierta agregación familiar, pero que solo el $5 \%$ tienen un carácter hereditario, siendo los más frecuentes el síndrome de cáncer de mama-ovario hereditario y el de cáncer de colon hereditario no polipósico o síndrome de Lynch ${ }^{4,26,27}$. Casi todos se trasmiten con un patrón autosómico dominante, pero se debe considerar también que la agrupación de varios cánceres de uno o varios tipos en una familia no siempre es debida a un carácter genético ${ }^{4}$ y que, en ocasiones, no seremos capaces de detectar la alteración, aun en presencia del riesgo ${ }^{26}$. A su vez, si tras una prueba genética positiva no se tiene clara y asequible la actuación consiguiente (prueba de cribado y/o diagnóstica, quimioprevención, cirugía profiláctica), solo se incrementará la angustia, sin poder dar respuesta a la necesidad creada tras la realización de la prue$\mathrm{ba}^{26-28}$.

Edad de aparición más temprana que en la población general

Alta incidencia de cáncer en la familia

Presencia del mismo tipo de cáncer en los miembros de una familia

Bilateralidad del tumor, cuando éste se presenta en órganos pares

Multifocalidad. Varios cánceres primarios en el mismo individuo.

Asociación con defectos del desarrollo: Asociación de tumores con sobrecrecimiento corporal generalizado o asimétrico, dismorfias, malformaciones congénitas o retraso mental.

Tabla 8. Características de sospecha del cáncer hereditario ${ }^{4,27,28}$ 
Una posibilidad en perspectiva sería que, en ocasiones, y dado que solo las personas portadoras de mutación tendrían un incremento del riesgo de presentar esta enfermedad, se podría recomendar la realización de una prueba de cribado solo a ellos (por ejemplo una colonoscopia anual), quedando el resto de familiares sometidos a un riesgo y necesidad de pruebas similar al de la población general ${ }^{26}$.

En la tabla 9 se presentan, de forma simplificada, los principales tipos de cáncer hereditario y sus criterios de estudio. especialmente cuidadosos en valorar los aspectos éticos de nuestras actuaciones y sus repercusiones. En este sentido recomendamos dos excelentes revisiones y reflexiones de Melguizo y de García Abellán, recientemente publicadas ${ }^{17,29}$.

En todas las actuaciones relacionadas con el consejo genético se deben tener en cuenta una serie de circunstancias que condicionan las ventajas o inconvenientes de realizar un estudio a una persona sana, como son: la existencia de una prueba suficientemente fiable y accesible, el conocimien-

Cáncer de mama, o de mama y ovario: dos o más casos en un mismo individuo o familia o diagnosticados antes de los 50 años

Cáncer de colon, recto o endometrio: dos o más casos en un mismo individuo o familia o diagnosticado antes de los 50 años o asociado a otros tumores del Síndrome de Lynch (ovario, estómago, páncreas, renoureteral...)

Cáncer de colon asociado a poliposis adenomatosa familiar

Cáncer gástrico: dos cánceres familiares de tipo difuso o uno antes de los 40 años o asociado a un cáncer de mama familiar de histología lobulillar

Síndrome MEN-1 o MEN-2: adenoma hipofisario, paratiroideo, neuroendocrinos, gastroenteropancreático, medular de tiroides, feocromocitoma

Melanoma: dos melanomas malignos en un individuo o familia o asociado a cáncer de páncreas Cáncer de próstata: uno antes de los 50 años, dos antes de los 60 o tres de cualquier edad en primero o segundo grado

Síndrome de Li-Fraumeni: carcinoma infantil suprarrenal o de plexos coroideos o asociación de al menos dos de entre sarcoma, mama premenopáusico, cerebral o leucemia

Retinoblastoma en un familiar

Cáncer renal familiar y leiomatosis

Cáncer basocelular: 2 o más o uno de ellos antes de los 30 años

Dobles neoplasias salvo asociadas a factores externos

Tabla 9. Tipos de cáncer hereditario más frecuentes y criterios de estudio genético ${ }^{3,4,27}$

\section{ASPECTOS ÉTICOS}

La información genética tiene un carácter único (no existen dos personas iguales y, además, no existe ningún otro dato médico tan nuestro como la información genética), es inalterable en el tiempo, escapa totalmente a nuestra voluntad, tiene capacidad predictiva $y$, por último, nos vincula de manera inseparable con el resto de los miembros de nuestra familia ${ }^{26}$. A su vez, el CG tiene importantes riesgos de vulneración de confidencialidad, alteración emocional, culpabilización y discriminación laboral y social ${ }^{17}$. Por dicho motivo, debemos ser to de la magnitud del riesgo, una información suficiente sobre la carga clínica, social, emocional y financiera que supondrá el resultado positivo, las posibilidades de actuar para prevenir o paliar los efectos de la enfermedad con mejores resultados que si se actúa después del diagnóstico clínico, y los eventuales desarrollos en el campo de dicha afección genética, que puedan incidir en futuros tratamientos específicos que mejoren la calidad de vida de los afectados ${ }^{7,16,30}$. Y, en todo caso, las pruebas genéticas siempre se deberán realizar en un marco garantizado de asesoramiento pre y post estudio. En este sentido, la LIB $^{20}$ regula los aspectos a tener en cuenta en cada una de las áreas de 
interés: indicación clara, información suficiente, consentimiento expreso y por escrito, derecho a la información o no información si así lo desea el probando, cualificación del personal, confidencialidad, conservación de datos, equidad y universalidad y calidad de las pruebas y de las prestaciones diagnósticas y terapéuticas ${ }^{20,31}$. Asimismo, expertos de la Comisión Europea recogen también 25 recomendaciones que constituyen una especie de "código de conducta" que rija la aplicación de los test genéticos y sirva de referencia a los responsables políticos de su regulación ${ }^{32}$.

Hay que tener en cuenta que la información proveniente de una prueba genética puede afectar a una familia entera. Tanto si se trata de un gen dominante desde un punto de vista genético, como si el individuo es solo portador, los padres, hijos, hermanos e incluso los parientes lejanos de esa persona podrían también verse afectados. ¿A quién se debe informar del resultado y quién debe ser el informante? ¿Cómo puede condicionar el riesgo de ser portador de un gen patógeno la elección de pareja? ¿A quién más debería realizarse la prueba? ${ }^{21}$. ¿Cómo se debe abordar el hallazgo de una incompatibilidad genética y de una falsa paternidad? ${ }^{32}$. ¿Qué consecuencias tiene la selección de un embrión con fines terapéuticos de un hermano, del que será potencial eterno donante? ${ }^{17}$. ¿Qué consecuencias y conflictos éticos plantea la posibilidad u obligación moral de que determinados datos sean conocidos por las empresas o por las compañías de seguros? ${ }^{29}$. Y, en el caso de un menor, ¿la decisión de la realización de la prueba, adoptada por los padres, vulnera su derecho de confidencialidad y debería ser él quien decidiera más adelante realizarse o no la prueba? Sucede también que, dado que se han inventado relativamente pocas intervenciones para mejorar el desenlace de la mayoría de los desórdenes genéticos, como por ejemplo en la corea de Huntington, el daño potencial puede ser sustancial y el beneficio nulo'

También es importante señalar que durante el asesoramiento genético ningún médico debe aconsejar a sus pacientes, sino que debe limitarse a aclarar conceptos y dudas e informarles adecuadamente para que ellos puedan tomar sus decisiones de forma libre y autónoma. Nuestro papel nunca será directivo, sino informativo y clarificador ${ }^{34}$.

Por supuesto, en todas las actuaciones relacionadas con el consejo genético, del mismo modo que en toda nuestra práctica profesional, están vigentes los principios básicos de la ética: beneficencia, no maleficencia, autonomía, justicia y confidencialidad. Pero existen otros principios que pueden entrar en conflicto y en los que es difícil, a veces, establecer prioridades: defensa de la vida (por ejemplo, en el caso de la selección de embriones siempre se producirá la eliminación de aquellos no elegidos y los donantes deberían conocer este hecho y el destino de los mismos ${ }^{17}$ ), igualdad de oportunidades o no discriminación, etc.

\section{CONCLUSIONES}

Las posibilidades de intervención del profesional de AP en el asesoramiento genético son amplias y variadas. No obstante, su papel deberá quedar limitado en la mayoría de las ocasiones a una labor de detección de situaciones de riesgo, consejo para la eliminación de potenciales agresores, orientación a los servicios correspondientes y labor de información y apoyo dentro de sus posibilidades. Puntualmente estará en su mano la posibilidad de aplicar una medida preventiva, indicar la realización de alguna prueba de cribado y, en su caso, las pruebas diagnósticas correspondientes.

Los aspectos éticos deben ser considerados cuidadosamente, ya que toda la información obtenida de las pruebas genéticas acabará siendo reflejada en la Historia Clínica y nos planteará múltiples dilemas en función de los derechos de los potenciales implicados.

En la tabla 10 se resumen las principales habilidades en el terreno de la genética que deberían formar parte del acervo curricular del profesional de Atención Primaria.

Para finalizar, recogemos una de las afirmaciones de la Declaración Universal sobre el Genoma Humano y los derechos humanos: "Las investigaciones sobre el genoma humano y sus aplicaciones abren inmensas perspectivas de mejoramiento de la salud de los individuos y de toda la humanidad, pero destacando que deben, al mismo tiempo, respetar plenamente la dignidad, la libertad y los derechos de la persona humana, así como la prohibición de toda forma de discriminación fundada en las características genéticas" 7,35 . 
Ser capaz de construir el árbol genealógico
Conocer los patrones básicos de la herencia
Identificar las principales enfermedades hereditarias
Conocer los principales cánceres con carácter hereditario
Identificar oportunamente los pacientes con riesgo de enfermedad hereditaria
Orientar sobre consejo genético preconcepcional o postconcepcional
Conocer las posibilidades técnicas, asistenciales, de información y de tratamiento
Saber derivar adecuadamente a las personas con riesgo
Ofrecer una información suficiente e inteligible a las personas con riesgo
Conocer los límites éticos y legales de las actuaciones en materia genética
Aplicar las actuaciones recomendadas en función del riesgo
Apoyar psicológica y asistencialmente al paciente y familiares

Tabla 10. Habilidades en consejo genético del Médico de Familia $3,4,33$

\section{BIBLIOGRAFÍA}

1. González-Lamuño D, García Fuentes M. Enfermedades de base genética. An Sist Sanit Navar. 2008;31(Supl-2):105126.

2. REAL DECRETO 1030/2006, de 15 de septiembre, por el que se establece la cartera de servicios comunes del Sistema Nacional de Salud y el procedimiento para su actualización. BOE núm. 222. Sábado 16 septiembre de 2006: 32650- 32679.

3. Ejarque I, García-Ribes M, Sorlí JV, Arenas E y Martín V. Papel de la atención primaria ante el cáncer hereditario. Aten Primaria. 2008;40(10):525-9.

4. Robles Díaz L (Coord.), Balmaña J, Garrell Lluis I, Grandes Velasco S, Graña Suárez B, Guillén Ponce C et al. Consenso en cáncer hereditario entre la sociedad española de oncología médica (seom) y sociedades de atención primaria (SEMFYC, SEMERGEN, SEMG). Med Gen y Fam (digital). 2012;1(7):333-342.

5. Miesfeldt S, Jameson JL. La genética en la práctica clínica. En: Harrison. Principios de Medicina Interna. México: McGraw-Hill Interamericana Ed; 2009. pp 414-20.

6. Palau Martínez F y Espinós Armero C. Consejo genético en las enfermedades neurológicas. FMC. 2009;16(2):92-102.

7. Santos Alcántara MJ. Aspectos bioéticos del consejo genético en la era del proyecto del genoma humano. Acta Bioethica. 2004; año X, № 2: 191-200.

8. García Ribes M, Verde González MP, Suerio Justel J, Rosado Bartolomé A, Sorlí Guerola JV. Abordando las enfermedades raras desde la consulta de Atención Primaria: si se quiere, se puede. Rev Clin Med Fam. 2013;6(1):32-6.

9. Alonso Cerezo C, Cañadas Gálvez MC, de la Fuente Hernández LA, García Ochoa C, García Sagredo JM et al. Recomendaciones para el estudio genético de la pareja con alteraciones en la reproducción. Rev Lab Clin. 2009;2(4):196-203.

10. García Ribes M. Genéticas: Raras y no tan raras. AMF. 2012;8(3):135-41.
11. Rueda JR, Briones Pérez de la Blanca E. Servicios de diagnóstico genético para las enfermedades hereditarias en España. Agencia de Evaluación de Tecnologías Sanitarias de Andalucía (AETSA), Sevilla, Octubre de 2002. Disponible en: http://www.juntadeandalucia.es/salud/servicios/ contenidos/nuevaaetsa/up/AETSA_P_2003_Tests_Geneticos ES IPTS.pdf [consultado el 12-02-2014]

12. Solari AJ. Genética Humana, Fundamentos y aplicaciones en Medicina. Buenos Aires: Médica panamericana; 2007. pp 173-7.

13. Oliva R, Margarit E, Milá M, Casademont J, Golomer D. Genética básica: Alteraciones moleculares y patrones de herencia. Jano. 2001: 719-1039.

14. Calvo Martín MT. Enfermedades genéticas, Consejo genético y Diagnóstico prenatal (Presentación). Disponible en: www.enferteruel.com/enferteruel/programas/deficiencias alcaniz/consejo genetico.ppt [consultado el 12-02-2014]

15. U.S. Preventive Services Task Force. Folic Acid for the Prevention of Neural Tube Defects: U.S. Preventive. Services Task Force Recommendation Statement. Ann Intern Med. 2009;150:626-631.

16. Márquez Calderón S, Castilla Alcalá JA, Briones Pérez de la Blanca E, Carriazo Pérez de Guzmán A. Guía para la toma de decisiones sobre incorporación de nuevas pruebas genéticas en el Sistema Nacional de Salud (Guía Gen). Agencia de Evaluación de Tecnologías Sanitarias de Andalucía. Madrid: Ministerio de Sanidad y Consumo, 2006.

17. Melguizo Jiménez M, Castro Martínez FJ, Orgaz Rosúa MJ. Genética y bioética: conflictos éticos en genética. AMF. 2013; 9(6):316-325.

18. Jefatura del Estado. Convenio para la protección de los derechos humanos y la dignidad del ser humano con respecto a las aplicaciones de la biología y la medicina. BOE núm. 251, 20 octubre de 1999: 36825-36830.

19. Jefatura del Estado. LEY 14/2006, de 26 de mayo, sobre técnicas de reproducción humana asistida. BOE núm. 126, 27 mayo de 2006: 19947-19956. 
20. Jefatura del Estado. LEY 14/2007, de 3 de julio, de Investigación biomédica. BOE núm. 159, 4 julio de 2007: 2882728848.

21. MacDonald Glenn L. El Uso de Pruebas Genéticas para Predecir Enfermedades: Implicaciones Éticas, Legales, y Sociales. Disponible en: http://www.actionbioscience.org/ esp/genomica/glenn2.html [consultado el 20-02-2014]

22. Abad Gimeno FJ, Pons Cabrera J, Micó Mérida M, Casterá Melchor DE, Bellés Medall MD, Sánchez Pedroche A. Categorías de riesgo de los medicamentos utilizados durante el embarazo. Guía rápida de consulta. Farmacia de Atención Primaria. 2005; 3(2):49-61.

23. Garrido Cuenca, N. A vueltas sobre la Prueba del Talón en Recién Nacidos. ¿Hacia un Programa Nacional de Cribado Neonatal Ampliado? Rev Clin Med Fam. 2012;5(31):1-2.

24. Jefatura del Estado. Ley $33 / 2011$, de 4 de octubre, General de Salud Pública. BOE núm. 240, 5 de octubre de 2011. 104593- 104626.

25. Ministerio de Sanidad, Política Social e lgualdad. Resumen ejecutivo del grupo de expertos sobre concreción de cartera común de servicios para cribado neonatal. Madrid, junio de 2013. Disponible en: http://www.msssi.gob.es/profesionales/saludPublica/prevPromocion/docs/ResumenEjecutivoCribadoNeonatal.pdf [consultado el 20-02-2014]

26. Pérez Segura P. Cáncer familiar y consejo genético. FMC. 2013;20(6):327-33

27. Salazar Sáez R. Indicaciones de estudios genéticos en Oncología. Consejo genético. Estrategias de prevención oncológica en portadores de alteraciones genéticas. Medicine. 2005;9(26):1732-5.

28. Cancelo Hidalgo MJ, López Parra M, Marcos González MV, Muñoz Algar MJ, Cancelo Hidalgo C y Álvarez de los Heros
Jl. Consejo genético en el cáncer de mama y ovario. ¿Y luego qué? Semergen. 2011;37(6):307-11.

29. Abellán García-Sánchez F. Los análisis genéticos dentro de la Ley de Investigación Biomédica. Revista de la Escuela de Medicina Legal. Junio de 2009: 22-42.

30. Junta de Andalucía. Plan de Genética de Andalucía. Líneas de acción. Disponible en: http://www.juntadeandalucia. es/salud/sites/csalud/galerias/documentos/c_1_c_6_planes estrategias/plan genetica/06 lineas.pdf [consultado el 20-02-2014]

31. Pérez Segura P. Los estudios genéticos y la Ley de Investigación Biomédica. Med Clin (Barc). 2009;132(4):154-156.

32. Mercadal M, Álvarez A, Hernández G, García-Algar O. Hallazgo inesperado en el consejo genético. An Pedia$\operatorname{tr}$ (Barc). 2013. Disponible en http://dx.doi.org/10.1016 /j.anpedi.2013.09.010

33. McNally E, Cambon-Thomsen A, Brazell C, Cassiman JJ, Kent A, Lindpaintner K. 25 recomendaciones sobre las repercusiones éticas, jurídicas y sociales de los tests genéticos. Luxemburgo, 2004; Oficina de Publicaciones Oficiales de las Comunidades Europeas. Disponible en http:// ec.europa.eu/research/conferences/2004/genetic/pdf/recommendations es.pdf. [consultado el 24-02-2014]

34. Declaración Universal sobre el Genoma Humano y los Derechos Humanos. UNESCO, París, 11 Noviembre de 1997. Consultado en http://portal.unesco.org/es/ev.php-URL ID=13177\&URL DO=DO TOPIC\&URL SECTION=201. html [consultado el 24-02-2014]

35. Ejarque I, García-Ribes M, Martín V. El médico de familia y sus competencias en genética clínica. Aten Primaria. 2007;39(3):113-4. 